\title{
CHEBYSHEV SUBSPACES OF FINITE CODIMENSION IN SPACES OF CONTINUOUS FUNCTIONS
}

\author{
A. L. BROWN
}

(Received 13 July 1976)

Communicated by E. Strzelecki

\begin{abstract}
A. L. Garkavi in 1967 characterized those compact metric spaces $X$ with the property that the space $C(X)$ of real-valued continuous functions possesses Chebyshev subspaces of fine codimension $\geqslant 2$. Here compact Hausdorff spaces with the same property are characterized in terms of certain standard subspaces of the space $[0,1] \times\{0,1\}$ equipped with a lexicographic order topology. Garkavi's result for metric spaces is exhibited as a corollary. The proof depends upon a simplification of a characterization by Garkavi of the Chebyshev subspaces of finite codimension in $C(X)$.
\end{abstract}

Subject classification (Amer. Math. Soc. (MOS) 1970): primary 41 A 65, 46 E 15; secondary 54 G 99.

Chebyshev subspaces of finite codimension in general Banach spaces and, in particular, in spaces of continuous functions were considered in the 1960's by, amongst others, R. R. Phelps and A. L. Garkavi. An account of much of that work can be found in the books of I. Singer (1970 and 1974) to which the reader can refer for definitions and references.

Throughout the discussion $X$ will denote a compact Hausdorff space and $C(X)$ the Banach space of real valued continuous functions on $X$.

Garkavi $(1964,1967)$ characterized the Chebyshev subspaces of finite codimension in $C(X)$ in terms of the annihilators in the dual space $C(X)^{*}$. In a subsequent paper (1967a) he obtained a simple characterization of those compact metric spaces $X$ with the property that $C(X)$ possesses Chebyshev subspaces of finite codimension greater than one.

The present paper (1) simplifies Garkavi's characterization of the Chebyshev subspaces of finite codimension in $C(X)$; (2) shows that if $C(X)$ possesses one Chebyshev subspace of finite codimension greater than one then it possesses Chebyshev subspaces of every finite codimension greater than one; and (3) characterizes, in terms of the space $[0,1] \times\{0,1\}$ equipped with a lexicographic order topology, those compact Hausdorff spaces $X$ for which such Chebyshev subspaces exist. Garkavi's result for metric spaces is exhibited as a corollary. 
The set of isolated points of $X$ will be denoted by $\Delta$. The dual space $C(X)^{*}$ of $C(X)$ will be identified with the space of regular Borel measures on $X$. If $\mu \in C(X)^{*}$, then $\mu^{+}, \mu^{-}$and $|\mu|$ will have their standard connotations, the support of $\mu$ will be denoted $s(\mu)$ and if $x \in X$ we will occasionally write $\mu(x)$ in place of $\mu(\{x\})$.

\section{Chebyshev subspaces}

Garkavi's characterization of the Chebyshev subspaces of finite codimension in $C(X)$ is as follows.

A closed linear subspace $M$ of finite codimension $n \geqslant 1$ in $C(X)$ is Chebyshev if and only if the annihilator $M^{\perp}$ of $M$ in $C(X)^{*}$ satisfies the four conditions:

(a) $s\left(\mu^{+}\right) \cap s\left(\mu^{-}\right)=\varnothing$ for each $\mu$ in $M^{\perp}$;

(b) $\mu$ is absolutely continuous with respect to $\nu$ on $s(\nu)$ for each $\mu$ and $\nu$ in $M^{\perp} \backslash\{0\}$

(c) $X \backslash s(\mu)$ has at most $n-1$ points for each $\mu$ in $M^{\perp} \backslash\{0\}$;

(d) if $r \leqslant n-1$ and $x_{1}, \ldots, x_{r}$ are distinct isolated points of $X$ then

$$
\left\{\left(\mu\left(x_{1}\right), \ldots, \mu\left(x_{r}\right)\right): \mu \in M^{\perp}\right\}=\mathbf{R}^{r} .
$$

It is a simple consequence of (a) and (c) that

(e) $s\left(\mu^{+}\right)$and $s\left(\mu^{-}\right)$are open and closed subsets of $X$ for each $\mu$ in $M^{\perp}$.

This characterization will be simplified by exploiting the ideas and arguments of Garkavi's subsequent paper (1967a).

THEOREM 1. Let $M$ be a closed linear subspace of finite codimension $n \geqslant 1$ in $C(X)$, let $\lambda_{1}, \ldots, \lambda_{n}$ be a basis of $M^{\perp}$ and $\lambda=\left|\lambda_{1}\right|+\ldots+\left|\lambda_{n}\right|$. If $M$ is a Chebyshev subspace of $C(X)$ then:

(1) $\lambda$ is a positive measure with $s(\lambda)=X$, and

(2) $M^{\perp}$ is of the form $M^{\perp}=\{f \lambda: f \in \tilde{M}\}$, where

(3) $\tilde{M}$ is an n-dimensional subspace of $C(x)$ such that for each $f \in \tilde{M} \backslash\{0\}$

$(\alpha)$ each $x \in X$ either is a local maximum of $f$ or is a local minimum of $f$,

(B) $\lambda\left(f^{-1}(0) \backslash \Delta\right)=0$, and

$(\gamma)$ if $r \leqslant n$ and $x_{1}, \ldots, x_{r}$ are distinct isolated points of $X$ then

$$
\left\{\left(f\left(x_{1}\right), \ldots, f\left(x_{r}\right)\right): f \in \tilde{M}\right\}=\mathbf{R}^{r} .
$$

Conversely, if $M^{\perp}$ is of the form (2) where conditions (1) and (3) are satisfied then $M$ is a Chebyshev subspace of $C(X)$.

Proof. Suppose that $M$ is a Chebyshev subspace of $C(X)$,so that conditions (a) to (e) are satisfied. It follows from (c) and (d) that $s(\lambda)=X$. (In particular an isolated point of $X$ has positive $\lambda$-measure and, therefore, the set $\Delta$ of isolated points is countable.) 
Let $\mu \in M^{\perp} \backslash\{0\}$. It follows from (e), applied to each of $\lambda_{1}, \ldots, \lambda_{n}$ that $X$ can be expressed as a union of not more than $2^{n}$ pairwise disjoint open and closed subsets of $X$ on each of which $\lambda$ is a linear combination of $\lambda_{1}, \ldots, \lambda_{n}$. Let $X$ be a non-empty intersection of one of these sets with $s\left(\mu^{+}\right)$or $s\left(\mu^{-}\right)$; it is open and closed. If attention is restricted to $X$ it may be supposed that both $\lambda$ and $\mu$ are positive and are members of $M^{\perp}$.

The next steps of the proof come directly from Garkavi (1967a). For $x \in \bar{X}$ let

and

$$
\mathscr{U}=\{U: x \in U, U \text { an open set of } \bar{X}\}
$$

$$
\mathscr{E}(U)=\{E: E \text { a Borel subset of } U, \lambda(E) \neq 0\} .
$$

Define $\bar{f}$ and $f$ by

$$
\begin{aligned}
& \bar{f}(x)=\inf _{\delta(U)} \frac{\mu(E)}{\lambda(E)}, \\
& f(x)=\sup _{\mathscr{\delta}(U)} \inf _{\delta(U)} \frac{\mu(E)}{\lambda(E)} .
\end{aligned}
$$

Then $\tilde{f}(x)$ and $f(x)$ are limits over the directed sets $\mathscr{U}$ and

$$
0 \leqslant \underline{f}(x) \leqslant \tilde{f}(x)<\infty
$$

(the final inequality being a consequence of the definition of $\lambda$ ). It will be shown that $\underline{f}(x)=\tilde{f}(x)$. Suppose that for some $k$ and every $U \in \mathscr{U}$

$$
\inf _{\delta(U)} \frac{\mu(E)}{\lambda(E)}<k<\sup _{\&(U)} \frac{\mu(E)}{\lambda(E)} .
$$

For each $U \in \mathscr{U}$ choose $E_{1}$ and $E_{2}$ in $\mathscr{E}(U)$ such that

$$
\frac{\mu\left(E_{1}\right)}{\lambda\left(E_{1}\right)}<k<\frac{\mu\left(E_{2}\right)}{\lambda\left(E_{2}\right)} \text {. }
$$

Then

so that

$$
(\mu-k \lambda)\left(E_{1}\right)<0<(\mu-k \lambda)\left(E_{2}\right)
$$

$$
s\left((\mu-k \lambda)^{-}\right) \cap U \neq \varnothing, \quad s\left((\mu-k \lambda)^{+}\right) \cap U \neq \varnothing .
$$

This implies that $x \in s\left((\mu-k \lambda)^{-}\right) \cap s\left((\mu-k \lambda)^{+}\right)$which contradicts (a) for the measure $\mu-k \lambda$ in $M^{\perp}$. This proves that $\left(^{*}\right)$ cannot hold for every $U \in \mathscr{U}$ for any $k$. In particular, $f(x)=\tilde{f}(x)$.

Define $f$ on $\not{X}$ by $f(x)=\underline{f}(x)=\bar{f}(x)$. The function $f$ is continuous and satisfies condition ( $\alpha$ ). If $x \in \bar{X}$ and $\varepsilon>0$ then by the preceding paragraph with $k=f(x)$ there exists $U \in \mathscr{U}$ such that either

$$
f(x) \leqslant \frac{\mu(F)}{\lambda(F)} \leqslant \sup _{\mathscr{\delta}(U)} \frac{\mu(E)}{\lambda(E)}<f(x)+\varepsilon \text { for all } F \in \mathscr{E}(U)
$$


or

$$
f(x) \geqslant \frac{\mu(F)}{\lambda(F)} \geqslant \inf _{\mathscr{S}(U)} \frac{\mu(E)}{\lambda(E)}>f(x)-\varepsilon \text { for all } F \in \mathscr{E}(U) .
$$

If $y \in U$ then in the first case

$$
f(x) \leqslant \underline{f}(y)=\bar{f}(y)<f(x)+\varepsilon
$$

and in the second case

$$
f(x) \geqslant \bar{f}(y)=\underline{f}(y)>f(x)-\varepsilon .
$$

The assertion is proved.

The next step is to show that $f$ is a Radon-Nykodym derivative of $\mu$ with respect to $\lambda$ on $X$. It has to be shown that for every closed subset $E_{0}$ of $\vec{X}$

$$
\mu\left(E_{0}\right)=\int_{E_{0}} f d \lambda
$$

Let $\varepsilon>0$. For each $x$ in $E_{0}$ there is an open neighbourhood $U_{x}$ of $x$ such that for all $y \in U_{x}$ and all $E \in \mathscr{E}\left(U_{x}\right)$

$$
\begin{aligned}
& f(x)-\varepsilon<f(y)<f(x)+\varepsilon, \\
& f(x)-\varepsilon<\frac{\mu(E)}{\lambda(E)}<f(x)+\varepsilon .
\end{aligned}
$$

Let $U_{x_{1}}, \ldots, U_{x_{m}}$ be a finite cover of $E_{0}$ and express $E_{0}$ as a disjoint union $E_{0}=\bigcup_{j=1}^{m} E_{j}$ with $E_{j}$ a Borel subset of $U_{x_{i}}$. Then

$$
\begin{aligned}
\left|\mu\left(E_{0}\right)-\int_{E_{0}} f d \lambda\right| & \leqslant \sum_{j=1}^{m}\left(\left|\mu\left(E_{j}\right)-f\left(x_{j}\right) \lambda\left(E_{j}\right)\right|+\left|f\left(x_{j}\right) \lambda\left(E_{j}\right)-\int_{E_{1}} f(x) d \lambda\right|\right) \\
& \leqslant 2 \varepsilon \lambda\left(E_{0}\right) .
\end{aligned}
$$

(Note that if $\lambda\left(E_{j}\right)=0$ then $\mu\left(E_{j}\right)=0$.) The inequality holds for every $\varepsilon>0$ and so the assertion is proved.

It now follows that $\mu=f \lambda$ for some $f \in C(X)$ satisfying condition (3)( $\alpha)$. Consequently, $M^{\perp}$ is of the form (2) with $\tilde{M}$ a linear subspace of $C(X)$. The measure $\lambda$ satisfies (1).

Condition (d) is now equivalent to the cases $r \leqslant n-1$ of condition (3) ( $\gamma$ ). If $f \in \tilde{M}$ then $(X \mid s(f \lambda)) \cap \Delta=f^{-1}(0) \cap \Delta$. The case $r=n$ of $(3)(\gamma)$ therefore follows from (c).

Now suppose that $f \in \tilde{M}$ and that $E$ is a Borel subset of $f^{-1}(0) \backslash \Delta$. Then $(f \lambda)(E)=\int_{E} f d \lambda=0$. Therefore, $|f \lambda|\left(f^{-1}(0) \mid \Delta\right)=0$. It follows by condition (b) that $f^{-1}(0) \backslash \Delta$ is $\mu$-null for every $\mu \in M^{\perp}$ and so, by the definition of $\lambda$, also $\lambda$-null. Therefore, $(3)(\beta)$ is satisfied.

Conversely, suppose that $M^{-1}$ is of the form (2) and that conditions (1) and (3) are satisfied. Conditions (a) - (d) must be verified. 
If $f \in \widehat{M}$ and $k$ is any number then by (3)( $\alpha$ ) and the continuity of $f$ the sets $\{x: f(x)<k\}^{-}$and $\{x: f(x)>k\}^{-}$are disjoint. Condition (a) follows from the case $k=0$.

It follows from the case $r=n$ of $(3)(\gamma)$ that $\Delta n f^{-1}(0)$ contains at most $n-1$ points. Also $X \backslash s(f \lambda) \subseteq f^{-1}(0)$ and therefore $(X \backslash s(f)) \backslash \Delta$ is an open set which is $\lambda$-null by (3) $(\beta)$ and so empty by (1). This establishes (c). Condition (d) is equivalent to the cases $r \leqslant n-1$ of $(3)(\gamma)$.

Now consider $f, g \in \tilde{M} \backslash\{0\}$. Suppose that $E$ is a closed subset of $X$, that $E$ is $(f \lambda)$-null and $E \subseteq s(f \lambda)$. Then $E \cap \Delta=\varnothing$ and $E \cap f^{-1}(0)$ is $\lambda$-null by (3) $(\beta)$. Therefore

$$
(g \lambda)(E)=\int_{E} g d \lambda=\int_{E \bigvee^{-1}(0)} g d \lambda=\int_{E \backslash \bigvee^{-1}(0)} \frac{g}{f}(f d \lambda)=0 .
$$

This establishes (b).

\section{Conditions for the existence of Chebyshev subspaces}

In this section, we first obtain a preliminary characterization of those $X$ for which $C(X)$ possesses Chebyshev subspaces of codimension $\geqslant 2$ and simultaneously show that if $C(X)$ possesses one such subspace then it possesses many.

THEOREM 2. The following conditions on a compact Hausdorff space $X$ which is not finite are equivalent:

(1) $C(X)$ possesses Chebyshev subspaces of every finite codimension;

(2) $C(X)$ possesses at least one Chebyshev subspace of finite codimension $\geqslant 2$;

(3) $C(X)$ possesses a Chebyshev subspace of codimension 2;

(4) There exists a positive measure $\lambda$ and a function $f_{0} \in C(X)$ such that

$(\alpha) s(\lambda)=X$

$(\beta)$ for each real number $t$ the set $f_{0}^{-1}(t)$ is the union of $a \lambda$-null set and at most one isolated point of $X$;

$(\gamma)$ each $x \in X$ either is a local maximum of $f_{0}$ or is a local minimum of $f_{0}$.

ProOF. (2) $\Rightarrow(3)$. It is a consequence of a theorem of Ewald, Larman and Rogers (1970) that each finite dimensional normed linear space possesses Chebyshev subspaces of dimension one. If $E$ is a normed linear space and $M$ is a Chebyshev subspace of finite codimension $n$ in $E$, then a simple calculation shows that corresponding to a one-dimensional Chebyshev subspace of the quotient space $E / M$ there is a Chebyshev subspace of $E$ that contains $M$ and is of codimension $n-1$ (see Cheney and Wulbert, 1969). The implication (2) $\Rightarrow(3)$ is therefore true of any normed linear space (it extends an observation of Grünbaum and Klee recorded in 
Klee (1969)). It is possible to prove it in the present context by a more elementary argument based upon Theorem 1 .

(3) $\Rightarrow(4)$. This implication is a straightforward deduction from the case $n=2$ of Theorem 1. Suppose that $M$ is a Chebyshev subspace of codimension 2 . Let $\lambda_{1}, \lambda_{2}$ be a basis of $M^{\perp}, \lambda=\left|\lambda_{1}\right|+\left|\lambda_{2}\right|$. Then the conditions of Theorem 1 are satisfied and $\lambda_{1}=f_{1} \lambda, \lambda_{2}=f_{2} \lambda$ for some $f_{1}$ and $f_{2}$ in $C(X)$ with the property

$$
1=\left|f_{1}\right|+\left|f_{2}\right| \text {. }
$$

By (1) and (3) of Theorem 1 the set $A=\left\{x: f_{2}(x)>0\right\}^{-}$is an open and closed subset of $X$. Let $f_{0}(x)=f_{1}(x)$ if $x \in A$ and $f_{0}(x)=-f_{1}(x)$ if $x \notin A$, so that $f_{0} \in C(X)$. If $0<t \leqslant 1$ then

$$
\begin{array}{r}
f_{0}^{-1}(t) \subseteq\left(f_{2}-\left(\frac{1}{t}-1\right) f_{1}\right)^{-1}(0), \\
f_{0}^{-1}(-t) \subseteq\left(f_{2}-\left(1-\frac{1}{t}\right) f_{1}\right)^{-1}(0) .
\end{array}
$$

It follows that $f_{0}$ satisfies (4).

$(4) \Rightarrow(1)$. Suppose that $\lambda$ and $f_{0}$ satisfy condition (4) and let $n$ be an integer $\geqslant 2$. Let $\tilde{M}$ be the set of functions of the form $p_{\circ} f_{0}$ where $p$ is an algebraic polynomial of degree $\leqslant n-1$. It is easily seen that (3) of Theorem 1 is satisfied so that $\tilde{M}$ defines a Chebyshev subspace of codimension $n$ in $C(X)$. The proof of Theorem 2 is complete.

The next step is to introduce certain topological spaces in terms of which it is possible to characterize those $X$ which satisfy the conditions of Theorem 2 .

Definition. Let $\Gamma_{0}$ be the set $([0,1] \times\{0,1\}) \backslash\{(0,0),(1,1)\}$ and let $p_{0}: \Gamma_{0} \rightarrow[0,1]$ and $j_{0}: \Gamma_{0} \rightarrow\{0,1\}$ be the projections onto the coordinate spaces. Let $\leqslant$ denote the lexicographic ordering on $\Gamma_{0}$ : that is, $\alpha \leqslant \beta$ if and only if either $p_{0}(\alpha)<p_{0}(\beta)$ or $p_{0}(\alpha)=p_{0}(\beta)$ and $j_{0}(\alpha) \leqslant j_{0}(\beta)$. Then $\Gamma_{0}$ is a compact Hausdorff space in the order topology.

Relevant properties of the compact Hausdorff space $\Gamma_{0}$ will be listed.

(i) The sets of the form $\left\{\alpha \in \Gamma_{0}:(s, 1) \leqslant \alpha \leqslant(t, 0)\right\}$ are a base of open and closed sets for the topology of $\Gamma_{0}$.

(ii) The space $\Gamma_{0}$ has no isolated points.

(iii) The projection $p_{0}: \Gamma_{0} \rightarrow[0,1]$ is continuous (as a real-valued function) and each point of $\Gamma_{0}$ either is a local maximum of $p_{0}$ or is a local minimum of $p_{0}$.

(iv) The subspace $\Gamma_{u}=\left\{\alpha \in \Gamma_{0}: j_{0}(\alpha)=1\right\}$ of $\Gamma_{0}$ is homeomorphic to the interval $[0,1)$ with a half-open interval topology.

(v) If $X$ is a separable metric space and $p: X \rightarrow \Gamma_{u}$ is continuous then $p(X)$ is a countable subset of $\Gamma_{u}$. 
ProOF of (v). The following argument comes directly from Garkavi (1967a). Suppose that $X$ is a separable metric space and that $p: X \rightarrow[0,1)$ is continuous with respect to the half-open interval topology corresponding to the topology of $\Gamma_{u}$. Then for each $x \in X$ the set $p^{-1}([p(x), 1))$ is a neighbourhood of $x$ in $X$. Let

$$
A_{n}=\left\{x \in X: B\left(x, \frac{1}{n}\right) \subseteq p^{-1}([p(x), 1))\right\},
$$

where $B(x, 1 / n)$ is the open ball in $X$ with centre $x$ and radius $1 / n$. Then $X=\bigcup_{n=1}^{\infty} A_{n}$. For each positive integer $n$ the set $A_{n}$ is separable. If $\left\{x_{k}: k=1,2, \ldots\right\}$ is a dense subset of $A_{n}$ then $A_{n} \subseteq \bigcup_{k=1}^{\infty} B\left(x_{k}, 1 / n\right)$ and $p$ is constant on each of the sets $A_{n} \cap B\left(x_{k}, 1 / n\right)$. This proves (v).

(vi) If $\Gamma$ is a closed subset of $\Gamma_{0}$ then $\Gamma_{0} \backslash \Gamma$ is a union of a family of pairwise disjoint non-empty open intervals of $\Gamma_{0}$, each of the form $\left\{\gamma \in \Gamma_{0}: \alpha<\gamma<\beta\right\}$, $\left\{\gamma \in \Gamma_{0}: \gamma<\beta\right\}$ or $\left\{\gamma \in \Gamma_{0}: \alpha<\gamma\right\}$ with $\alpha$ and $\beta$ in $\Gamma$. These will be referred to as the complementary intervals of $\Gamma$, and the points $\alpha$ and $\beta$ as the end points.

Definition. A subspace $\Gamma$ of $\Gamma_{0}$ will be called an $R$ CI-subspace of $\Gamma_{0}$ if it is a closed subset of $\Gamma_{0}$, contains the point $(1,0)$ (a convenient normalization) and has the property that if $\alpha \in \Gamma$ is the end point of a complementary interval of $\Gamma$ then $j_{0}(\alpha)=0$. Thus RCI-subspaces of $\Gamma_{0}$ are ones for which the set of Right-hand end points of Complementary intervals of $\Gamma$ coincides with the set of Isolated points of $\Gamma$.

(vii) If $\Gamma$ is an RCI-subspace of $\Gamma_{0}$ then there exists a unique regular Borel measure $\lambda_{\Gamma}$ on $\Gamma$ such that, for all $\alpha \in \Gamma$ with $j_{0}(\alpha)=0$,

$$
\lambda_{\Gamma}(\{\gamma \in \Gamma: \gamma \leqslant \alpha\})=p_{0}(\alpha) \text {. }
$$

Its support is $\Gamma$. (The measure on $\Gamma_{0}$ can be thought of as a kind of Lebesgue measure: its image under $p_{0}$ is Lebesgue measure on $[0,1]$. For a general $\Gamma$ the measure $\lambda_{\Gamma}$ is obtained by sweeping the measure on a complementary interval onto the isolated right-hand end point.)

ProOF OF (vii). Let $\chi_{\alpha}$ denote the characteristic function of $\{\gamma \in \Gamma: \gamma \leqslant \alpha\}$. Then $\left\{\chi_{\alpha}: \alpha \in \Gamma, j_{0}(\alpha)=0\right\}$ is a linearly independent subset of $C(\Gamma)$ and, because $\Gamma$ is an RCI-subspace of $\Gamma_{0}$, the set separates the points of $\Gamma$. The set $A$ of linear combinations of these characteristic functions is a subalgebra of the algebra $C(\Gamma)$ and so, by the Stone-Weierstrass theorem, is dense in $C(\Gamma)$. There exists a unique linear functional $\lambda_{\Gamma}$ on $A$ such that $\lambda_{\Gamma}\left(\chi_{\alpha}\right)=p_{0}(\alpha)$, this linear functional extends by continuity to $C(\Gamma)$ and the extension is unique.

The final theorem can now be stated. 
THEOREM 3. A necessary and sufficient condition on an infinite compact Hausdorff space $X$ in order that $C(X)$ possess Chebyshev subspaces of finite codimension $\geqslant 2$ is that there exist a positive measure $\lambda$ on $X$, an RCI-subspace $\Gamma$ of $\Gamma_{0}$ and a continuous surjection $p: X \rightarrow \Gamma$ such that

(1) $s(\lambda)=X$,

(2) $\lambda_{\Gamma}$ is the image under $p$ of the measure $\lambda$, and

(3) $p^{-1}(\gamma)$ is a single point for each isolated point $\gamma$ of $\Gamma$.

If $X$ has no isolated points and the condition is satisfied then $\Gamma=\Gamma_{0}$. The condition is satisfied by any space $X$ that is an RCI-subspace of $\Gamma_{0}$.

Proof. It must be shown that the condition is equivalent to (4) of Theorem 2. Suppose that $X, \lambda$ and $f_{0}$ satisfy (4). It may be supposed that $\lambda(X)=1$. It follows from (4) $(\beta)$ and (4) $(\gamma)$ that for any number $k$ the sets $\left\{x: f_{0}(x)<k\right\}^{-}$and $\left\{x: f_{0}(x)>k\right\}^{-}$are disjoint open and closed subsets of $X$, together containing all but at most one point of $X$. Define $p: X \rightarrow \Gamma_{0}$ by

$$
p(x)= \begin{cases}\left(\lambda\left(\left\{y: f_{0}(y)<f_{0}(x)\right\}\right), 0\right) & \text { if } x \in\left\{y: f_{0}(y)<f_{0}(x)\right\}^{-}, \\ \left(\lambda\left(\left\{y: f_{0}(y) \leqslant f_{0}(x)\right\}\right), 1\right) & \text { if } x \in\left\{y: f_{0}(y)>f_{0}(x)\right\}^{-}, \\ \left(\lambda\left(\left\{y: f_{0}(y) \leqslant f_{0}(x)\right\}\right), 0\right) & \text { if } x \in \Delta\end{cases}
$$

and let $\Gamma=p(X)$.

It will be shown that $p: X \rightarrow \Gamma_{0}$ is continuous, that $\Gamma$ is an RCI-subspace of $\Gamma_{0}$ and that conditions (2) and (3) of the theorem are satisfied. The final statements of the theorem are straightforward. The verifications are elementary and unfortunately a little tedious.

Consider an $x \in X$ such that $x \in\left\{y: f_{0}(y)>f_{0}(x)\right\}^{-}$. Let $\varepsilon>0$. By the countable additivity of $\lambda$ there exists a positive integer $n$ such that

$$
\lambda\left(\left\{y: f_{0}(y) \leqslant f_{0}(x)+\frac{1}{n}\right\}\right)<\lambda\left(\left\{y: f_{0}(y) \leqslant f_{0}(x)\right\}\right)+\varepsilon .
$$

Let $U$ be an open subset of $X$ such that

and, for all $z \in U$,

$$
x \in U \subseteq\left\{y: f_{0}(y)>f_{0}(x)\right\}^{-}
$$

$$
f_{0}(x)+\frac{1}{n}>f_{0}(z) \geqslant f_{0}(x)
$$

If $z \in U$ then either $f_{0}(z)=f_{0}(x)$ or $f_{0}(z)>f_{0}(x)$. By $(4)(\beta)$ the second case must occur. In the first case $p(z)=p(x)$. In the second case $\left\{y: f_{0}(z)>f_{0}(y)>f_{0}(x)\right\}$ is open and non-empty and so, by (4) $(\alpha)$, is of positive $\lambda$-measure, and therefore

$$
p_{0}(p(x))+\varepsilon \geqslant p_{0}(p(z))>p_{0}(p(x)) \text {. }
$$


Therefore in both cases

$$
\left(p_{0}(p(x))+\varepsilon, 1\right) \geqslant p(z) \geqslant p(x) .
$$

This proves that $p$ is continuous at $x$ and that $p(x)$ is not an isolated point of $\Gamma$.

It can be shown in the same way that $p$ is continuous at points $x$ such that $x \in\{y: f(y)<f(x)\}^{-}$. It follows that $p$ is continuous and that $\Gamma$ is a closed subset of $\Gamma_{0}$.

It follows from (4) $(\beta)$ that $p(x)=(1,0) \in \Gamma$ for some point $x$ at which $f_{0}$ attains its maximum. In order to prove that $\Gamma$ is an RCI-subspace of $\Gamma_{0}$ it must be shown that if $x \in\left\{y: f_{0}(y)>f_{0}(x)\right\}^{-}$then $p(x)$ is not the end point of a complementary interval of $\Gamma$. As it has been shown that such a $p(x)$ is not an isolated point of $\Gamma$ it cannot be the left-hand end point of a complementary interval. If $f_{0}^{-1}\left(f_{0}(x)\right)$ contains an isolated point of $X$ then $\left(p_{0}(p(x)), 0\right) \in \Gamma$ and $p(x)$ is not the right-hand end point of a complementary interval of $\Gamma$. If $f_{0}^{-1}\left(f_{0}(x)\right)$ contains no isolated point of $X$ then, by (4) ( $\beta$ ), it is $\lambda$-null and

$$
p_{0}(p(x))=\lambda\left(\left\{y: f_{0}(y) \leqslant f_{0}(x)\right\}\right)=\lambda\left(\left\{y: f_{0}(y)<f_{0}(x)\right\}^{-}\right) .
$$

If $f_{0}$ attains its maximum on $\left\{y: f_{0}(y)<f_{0}(x)\right\}^{-}$at an isolated point $x^{\prime}$ then $p\left(x^{\prime}\right)=\left(p_{0}(p(x)), 0\right)$; if $f_{0}$ attains its maximum on $\left\{y: f_{0}(y)<f_{0}(x)\right\}-$ at $x^{\prime}$, but not at any isolated point, then again, by another appeal to $(4)(\beta), p\left(x^{\prime}\right)=\left(p_{0}(p(x)), 0\right)$. This proves that $p(x)$ is in no case an end point of a complementary interval of $\Gamma$.

To prove that (2) is satisfied consider an $x$ such that $j_{0}(p(x))=0$. If $x$ is isolated then

$$
p^{-1}(\{y: p(y) \leqslant p(x)\})=\left\{y: f_{0}(y)<f_{0}(x)\right\}^{-} \cup\{x\}
$$

and by $(4)(\beta)$, the set on the right differs by a $\lambda$-null set from $\left\{y: f_{0}(y) \leqslant f_{0}(x)\right\}$. If $x$ is not isolated then

$$
p^{-1}(\{y: p(y) \leqslant p(x)\})=\left\{y: f_{0}(y)<f_{0}(x)\right\}^{-}
$$

and the set on the right differs by a $\lambda$-null set from $\left\{y: f_{0}(y)<f_{0}(x)\right\}$. In both cases

$$
\lambda\left(p^{-1}(\{y: p(y) \leqslant p(x)\})\right)=p_{0}(p(x)) .
$$

Condition (2) now follows from (vii). Condition (3) clearly is satisfied.

Conversely, suppose that $X, \lambda, p$ and $\Gamma$ satisfy the stated conditions. Let $f_{0}=p_{0} \circ p$. It is now immediate that (4) of Theorem 2 is satisfied.

The corollaries of Theorems 2 and 3 include two results of Garkavi.

Corollary 1 (Garkavi (1967)). If $\Delta$ is countably infinite and dense in $X$ then $C(X)$ possesses Chebyshev subspaces of every finite codimension. 
Proof. Let $x_{n}, n=1,2, \ldots$, be the distinct isolated points of $X$. The condition of Theorem 3 is easily seen to be satisfied by a $\lambda$ and a $p$ with

$$
\lambda\left(x_{n}\right)=\frac{1}{n}-\frac{1}{n+1}, \quad p\left(x_{n}\right)=\left(\frac{1}{n}, 0\right) .
$$

Corollary 2. If $\Delta$ is not dense in $X$ then necessary conditions for $X$ to possess Chebyshev subspaces of finite codimension $\geqslant 2$ are:

(1) $\Delta$ is countable,

(2) there is a continuous surjection $q:\left(X \backslash \Delta^{-}\right)^{-} \rightarrow \Gamma_{0}$.

Proof. The necessity of (1) has been remarked and was well known. The necessity of (2) is a consequence of Theorem 3 applied to $\left(X \backslash \Delta^{-}\right)^{-}$: for if $C(X)$ possesses Chebyshev subspaces of finite codimension $\geqslant 2$ then $X$ satisfies (4) of Theorem 2, and so then does $\left(X \backslash \Delta^{-}\right)^{-}$, and it has no isolated points.

COROllary 3 (Garkavi (1967a)). If $X$ is an infinite compact metric space then $C(X)$ possesses Chebyshev subspaces of finite codimension $\geqslant 2$ if and only if the set $\Delta$ of isolated points of $X$ is dense in $X$.

Proof. The result follows from Corollaries 1 and 2, and property (v) of the space $\Gamma_{0}$.

This paper was written, and the work presented in it, was completed while the author enjoyed the relative freedom of a Draper Visiting Lectureship at the University of Newcastle, New South Wales. The University's hospitality is gratefully acknowledged.

\section{References}

E. W. Cheney and D. E. Wulbert (1969), "Existence and unicity of best approximations", Math. Scand. 24, 113-140.

G. Ewald, D. G. Larman and C. A. Rogers (1970), "The directions of the line segments and of the $r$-dimensional balls on the boundary of a convex body in euclidean space", Mathematika 17, 1-20.

A. L. Garkavi (1964), "Approximation properties of subspaces with finite defect in the space of continuous functions", Dokl. Akad. Nauk SSSR 155, 513-516. Translation: Soviet Math., Doklady 5, 440-443.

A. L. Garkavi (1967), "The Helly problem and best approximation in spaces of continuous functions", Izv. Akad. Nauk SSSR, Ser. Mat. 31, 641-656.

A. L. Garkavi (1967a), "On compact metric spaces which possess Chebyshev systems of measures", Mat. Sbornik (new ser.), 74, 209-217.

V. L. Klee (1969), "Can the boundary of a $d$-dimensional convex body contain segments in all directions?" Amer. Math. Month. 76, 408-410. 
I. Singer (1970), Best Approximation in Normed Linear Spaces by Elements of Linear Subspaces (Springer-Verlag, Berlin).

I. Singer (1974), The Theory of Best Approximation and Functional Analysis (CBMS, SIAM, Philadelphia).

University of Newcastle upon Tyne and University of Newcastle

England New South Wales, Australia 